\title{
Application of RFID and Video Imaging on Evacuation Observations in Offices and Public Buildings
}

\author{
TUOMO RINNE ${ }^{1}$, SIMO HOSTIKKA ${ }^{1}$, TUOMAS PALOPOSKI ${ }^{1}$, TIMO KORHONEN ${ }^{1}$, \\ JUHA-MATTI SAARI ${ }^{1}$, and SIMO HELIÖVAARA ${ }^{2}$ \\ ${ }^{1}$ VTT Technical Research Centre of Finland, P.O. Box 1000, FI-02044 VTT, Finland \\ ${ }^{2}$ Helsinki University of Technology, P.O. Box 1000, FI-02015 TKK, Finland
}

\begin{abstract}
In this work, two different types of evacuation situations were studied in order to provide validation data for some aspects of the evacuation modeling. The first type was evacuation drills which are normally carried out as part of the safety training of the staff in public buildings and offices. The second type was actual evacuations which occur every now and then. The main techniques used for the observation of evacuation events were video cameras, Radio Frequency Identification (RFID), and surveillance cameras. A large amount of information was obtained and the problems in the application of the observation techniques were identified. In particular, the results show that when the RFID technique is used, the placement of the antennas and tags is very important. With careful placement of the antennas and tags, the reliability of the RFID technique as applied in the current work may be sufficient for scientific purposes. In the observation of an actual evacuation of a large shopping centre, the recordings of the surveillance cameras were used to measure the flow rates of people. The results are very promising and indicate that the collection of surveillance camera recordings from large evacuations should be started.
\end{abstract}

KEYWORDS: evacuation, human behavior, Radio Frequency Identification, RFID, surveillance cameras, escape monitoring, validation.

\section{INTRODUCTION}

Traditionally, the design of evacuation capacity is based on the simple design criteria on the required width and length of evacuation routes. These criteria are based mainly on experimental findings and lessons learned from the past hazards. However, it would not be possible to build the large multi-purpose centres using the simple design rules. Instead, these places are usually designed using the performance based design method, in which the safety of the design is studied from the viewpoint of the entire system, not as fulfillment of individual rules given by the building code. The performance based design relies strongly on the numerical modelling and simulation of both the fire and evacuation processes.

For the reliability of the performance based design, simulation tools must be validated for the given type of application. In validation work, the computational results are usually compared against experimental results. Accurate and well documented measurements are needed in order to draw a good picture on the model strengths and weaknesses. Usually the primary technique of observations in evacuation experiments is video recording. The quantitative measurements from the video recordings are then made manually as a post-processing. The validation of decision making processes introduces a new challenge for the experimental design and measurements since quantitative techniques to observe human decisions, like the selection of escape routes, are not readily available. New techniques that can identify and monitor the location and movement of individuals are thus needed. One such a technique is the Radio Frequency Identification (RFID), commonly used for remote identification of people and items. The aim of this study is to provide validation data for some aspects of the evacuation modeling and to study the applicability of RFID on monitoring evacuees in field scale evacuation experiments.

\section{EXPERIMENTAL TECHNIQUES}

\section{Radio frequency identification (RFID)}

In recent years, radio frequency identification (RFID) methods for item management have developed rapidly. The frequencies used in these circumstances extend from $135 \mathrm{kHz}$ to $2.45 \mathrm{GHz}$. The applications can be divided to proximity, vicinity and remote sensing applications depending on the frequency. Frequency range from $433 \mathrm{MHz}$ to $2.45 \mathrm{GHz}$ and above is considered to be the remote sensing range. The 
techniques used in item management can easily be transferred into person identification. Most promising frequency ranges are the $869 \mathrm{MHz}$ (ultra high frequency, UHF) and $2.5 \mathrm{GHz}$ frequencies.

Both active and passive tags are available in the UHF-band. Active tags include a battery or other source of energy and are bigger and heavier than passive tags. Passive tags consist only of an antenna structure and a small $(\sim 1 \mathrm{~mm} \times 1 \mathrm{~mm})$ silicon chip. The antenna structure can be manufactured on a plastic sheet or board and the chip is glued or bonded on it. Passive tags receive the energy they need wirelessly from the reader device during the reading operation. Because of the popularity and a Finnish manufacturer, Raflatac (formerly Rafsec), passive EPC-tags were chosen for this study.

The UHF-readers chosen for this work were the FEIG ID ISC LRU 1000 and the Deister UDL 500. The FEIG reader has four external passive antennas while the Deister reader only has one internal antenna. Due to the fact that the FEIG reader antennas are passive, the maximum cable length from one antenna to the reader is limited to less than 10 meters, if a reading distance of 2 to 3 meters from the antenna is desired. Shorter cables give larger reading distance because the signal is not attenuated as much as in longer cables.

\section{Digital video cameras}

Video imaging may be used for two primary objectives in evacuation experiments: It may provide a recording of the door or exit flow for later use, or it may provide information on the reaction and premovement times, decision making processes and other human behaviour aspects for later analysis. The main difficulty of using the video cameras is related to the placement of cameras. This is especially true for indoor situations when the camera must be used without an operator. Custom made stands and supports must be used.

When video cameras are used as a recording of an exit flow, a proper technique is needed for counting the humans. The resulting data should contain the cumulative sum of evacuees at sufficient time resolution to enable the computation of flow rates. In this work, the flows were counted manually by using a custom made computer program, Evaccounter, that stores the times when the user presses the keyboard while watching the video. In this work, the key-presses were typically events when a single person passed a virtual control line shown in video e.g. a doorway or some other object. The MATLAB based software gives the exact times of key-presses and can scale the times based on the specified starting and ending times for low-speed video playback.

For monitoring the evacuation drills, we used the normal consumer level digital video cameras (mini-DV) with 1.3 Mpixel resolution. The videos were later on edited using commercial video editing software. In order to count the number of evacuees after the fire alarm, the videos were synchronized so that they all started one minute before the alarm.

\section{Surveillance cameras}

Many public buildings are nowadays equipped with surveillance cameras for security purposes. In shopping centers, for instance, the whole public space may be monitored by the security personnel. The modern surveillance camera systems have digital storage of the video material, and the video material from each camera can be viewed and processed afterwards. Typically the storage time of the video material is order of a few weeks. The utilization of such a video material was tested to find out the feasibility of surveillance cameras in research purposes.

\section{EXPERIMENTAL SETUP}

\section{Public library}

The evacuation of the main library of Helsinki University of Technology was carried out as part of the safety training program of the library staff in late winter. The staff was informed that the evacuation would take place in the given day. The library visitors were notified on the evacuation exercise by printed notes on the entrance doors. Exact time was not specified, nor were the details of the evacuation. A sketch of a floor plan and the numbering of the library doors during the evacuation are shown in Fig. 1. The visitors normally use doors 2 and 6. The staff also uses door 1 . Door 3 is for transportation of goods and is rarely used. Doors 4, 5 and 7 are only used for evacuations. 
It was anticipated that most staff members would exit through door 1, so two different RFID techniques were used at door 1 . Before the evacuation, a group of 33 people of the library staff were equipped with two different types of RFID tags. Immediately before the evacuation, researchers entered the building to make observations on crowd behaviour and outside the building to observe all doors. Video cameras were used to observe the evacuation from doors 1 and 4 . The evacuation drill started by activating the cold smoke generator, which located on the floor of the main lobby.

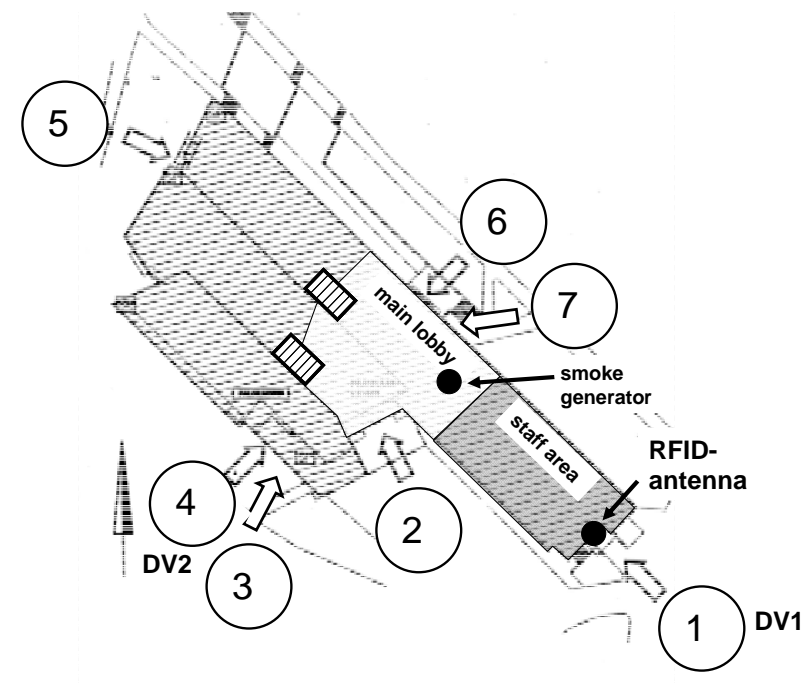

Fig. 1. The Main Library of Helsinki University of Technology.

\section{Large office building}

The second evacuation experiment was carried out in a large office building in Helsinki in November. The experiment differed from the library case mainly in terms of building geometry and number of the staff regularly working. The building has 7 floors and 4 entrances from the street level and normally, there are about 500 employees working in the building. The office building is illustrated in Fig. 2, where the entrances are also shown. The door 1 is normally used as a main entrance of the building for staff and visitors. Doors 2 and 3 are used by the employees only, and lead from the street level to staircases, where the elevators and stairs can be used to access the upper floors. Door 4 is used as an exit in evacuation situations. There is an access from staircase 3 to staircase 2 through the first floor offices. This access is shown by the upward dashed arrow of staircase 3 in Fig. 2.

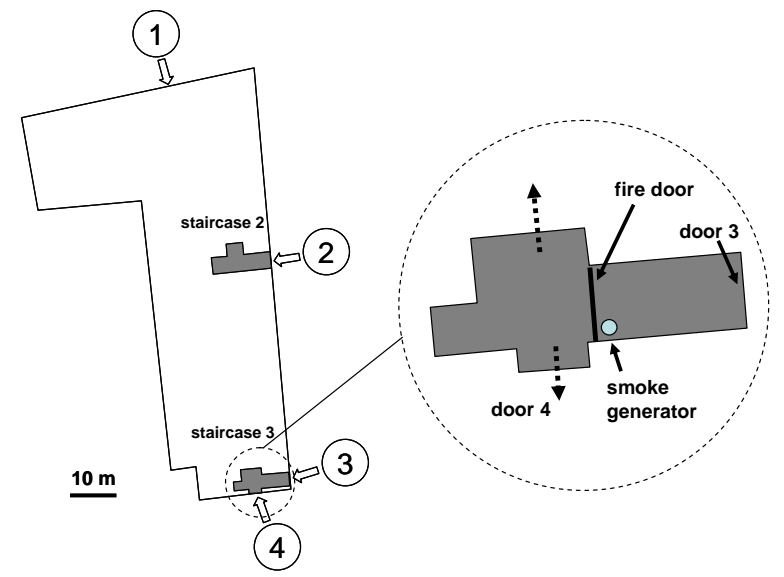

Fig. 2. The geometry of large office building showing the 4 entrances. The gray areas present the staircases at the street level and the dashed arrows present the exit routes from the staircase 3. 
The evacuation was carried out as part of the safety training program of the building employees. The evacuation was planned by the fire researcher team, local safety organisation, and local fire brigade. The main idea of the exercise was to force the evacuees to use an escape route that was unusual and not used in daily basis. This was realized by blocking the entrances 1 and 3 (see Fig. 2) with cold smoke generators, which were operated manually by the safety organisation personnel. The generators were placed at the street level inside entrances 1 and 3. The smoke generator in staircase 3 was placed between the outer door (door 3) and a fire door, so that people could still descend safely using the stairs to the street level behind the fire door and then either walk inside the building to staircase 2 and the door 2 in, or use the door 4 for the evacuation, see Fig. 2.

The observation focused the staircase 2. The staircase was equipped with six mini-DV cameras and five RFID antennas. For RFID, the FEIG reader with four antennas was assembled into the staircase. The antennas were placed on the half landing facing down towards the descending test persons at a distance of approximately 2-3 meters. Antenna number 1 was between floors 6 and 5, number 2 between floors 5 and 4 and so on. The Deister reader was placed at street level (floor 1 ) in front of the door 2 . The reader was facing down approximately 1 meter above the evacuating people. For RFID, four groups of people were chosen from the building. The groups were equipped with ID badges with colour code and a tag inside. Written instructions of correct attachment of the ID badge were given to each test person.

\section{Medium sized office building}

The third experiment was organized in a medium sized office located in Helsinki. Normally, about 300 people work in the building. The building has 4 floors and 5 exits described in Fig. 3 . The door 5 is the main entrance to the building and the other 4 doors are used only for evacuation purposes. Doors 1-3 lead to an inner court of the building, from where the archway leads to the street. Door 4 leads straight to the archway.

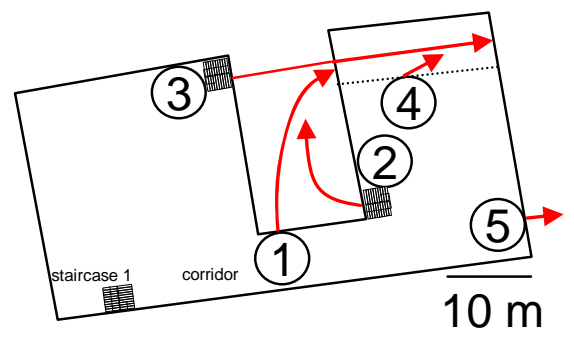

Fig. 3. Medium sized office building having 5 evacuation routes illustrated with arrows.

The evacuation was carried out in November as part of the safety training program of the building employees. The evacuation was planned by the fire researcher team and the local safety organisation. The main idea of the exercise was to force the evacuees to use an escape route that was unusual and not used in daily basis. This was realized by blocking the internal stairway leading to main entrance (door 5) with cold smoke. It was anticipated that most people from upper floors would then use the route through staircase 1 , corridor at the street level, and Door 1. The FEIG reader with four antennas was assembled into the staircase 1.

The DV-cameras were mainly used for recording the events of the internal corridor at the street level. Cameras were placed in both ends of the corridor and one camera to record the doorway of staircase 1 . Doorway camera recorded also the events through the glazing near the door inside the staircase 1 . Cameras were not placed inside the staircase 1 . Outside the building, one camera per door was used ( 5 cameras in all) and one camera was placed in a car park at the basement. The outside cameras were operated manually. Cameras inside the building were alone and they were started about 5 min before the alarm. 


\section{RESULTS AND DISCUSSION}

\section{Public library}

\section{General observations}

The evacuation started at one o'clock in the afternoon. A smoke generator was put in operation in the lobby, thus preventing the use of doors 2 and 6 . A fire alarm went off 37 seconds after the smoke generator was started, and evacuation began. The alarm signal was a loud bell sound. Soon after the alarm, the staff members that were part of the safety organization started to lead people towards the evacuation doors. In only few seconds after the alarm, the smoke started to flow up one of the main stairways. In 5 minutes 52 seconds after the fire alarm, all 189 people, that had been in the building, had evacuated.

After the fire alarm, the people in the second floor had to choose between three possible routes: main stairway and two evacuation doors through the internal stairways. The smoke filled the main stairway very soon after the alarm and made it impossible to use. In that situation, a great majority of people decided to evacuate through the stairway and door number 5 , which was in the opposite end of the building to the 'fire'. During the evacuation, people clearly followed the majority of the crowd to the door 5, even though they had to wait some time the access to the stairway. The staff members tried to give instructions and distribute people evenly to the two available stairways but the many evacuees did not pay much attention to these instructions.

The alarm signal was so loud and clear, that we can assume all the people observed the fire at the moment of fire alarm. After the alarm, people started to collect their things, pack the bags and wear jackets, because the exercise was held in winter time. Some people did all that in just a few seconds, some spent almost two minutes.

\section{Quantitative results}

The premovement times of 42 people, i.e. the time from alarm to the actual movement towards an exit, were collected from the videos. The average premovement time was $36 \mathrm{~s}$. The cumulative distribution of these times is shown in Fig. 4, accompanied by the LMS-fitted Weibull and LogNormal distributions. Comparing our result to a larger amount of data, we can see that the average values for premovement time are in range of 19-83 s [1, 2, 3].

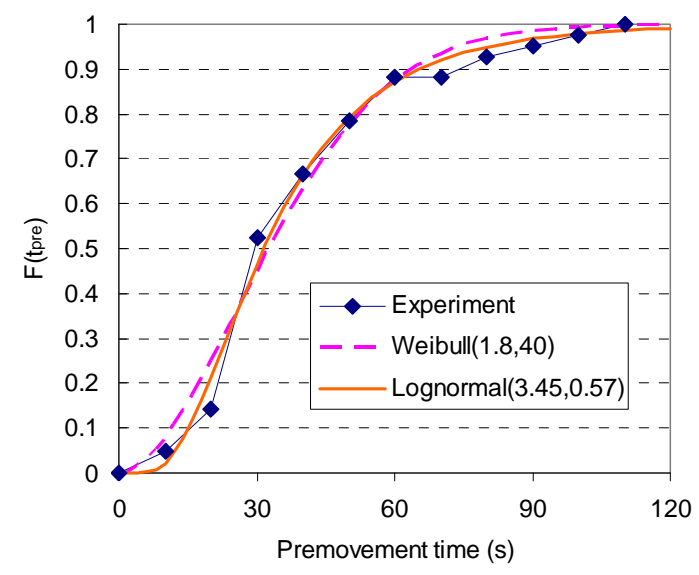

Fig. 4. Distribution of observed premovement times $(\mathrm{N}=42)$ in the public library and fitted Weibull and LogNormal distributions.

The cumulative number of evacuated people for each door is shown in Fig. 5. Most staff members used the door 1 and the most visitors used the door 5 . The flow of people out of the door 5 was quite steady from about $70 \mathrm{~s}$ to $200 \mathrm{~s}$ from the alarm, and probably controlled by the width of the stairway and the doors leading to the stairway. It was also noteworthy that evacuees coming outside from the door 5 had to walk in 
narrow path formed in snow bank - suggestion that the snow would be removed also from the front of emergency exit doors.

The flow rate $J$ of the door 5 was found to be about 0.7 persons per second, which is, stated in a form of the specific flow $J_{s}$, about 0.8 persons $\cdot \mathrm{s}^{-1} \cdot \mathrm{m}^{-1}$. However, the flow rate is based on the manual bookkeeping, and the uncertainty of the actual flow rate is quite high. The flow rate $J$ and the specific flow $J_{S}$ are determined by Eqs. (1) and (2),

$$
\begin{aligned}
& J=\frac{\Delta N}{\Delta t}, \\
& J_{s}=\frac{\Delta N}{\Delta t \cdot w},
\end{aligned}
$$

where $\Delta N(-)$ is the number of evacuated people within a time interval $\Delta t(\mathrm{~s})$ and $w(\mathrm{~m})$ is the width of the door.

The library evacuation was used for preliminary testing of the different technical monitoring techniques. Both inductive and UHF RFID antennas were installed at door 1. A comparison of the video and technical observations revealed that the inductive RFID observed and identified 5 of 12 people and the UHF RFID observed and identified only 3 of 12 . The main reason for the low identification percentage was that the tags were placed close to the body or some other electrically conducting object, where the electromagnetic field ceased to exist. Test persons who were identified had the tags on their clothing or otherwise far away from their body.

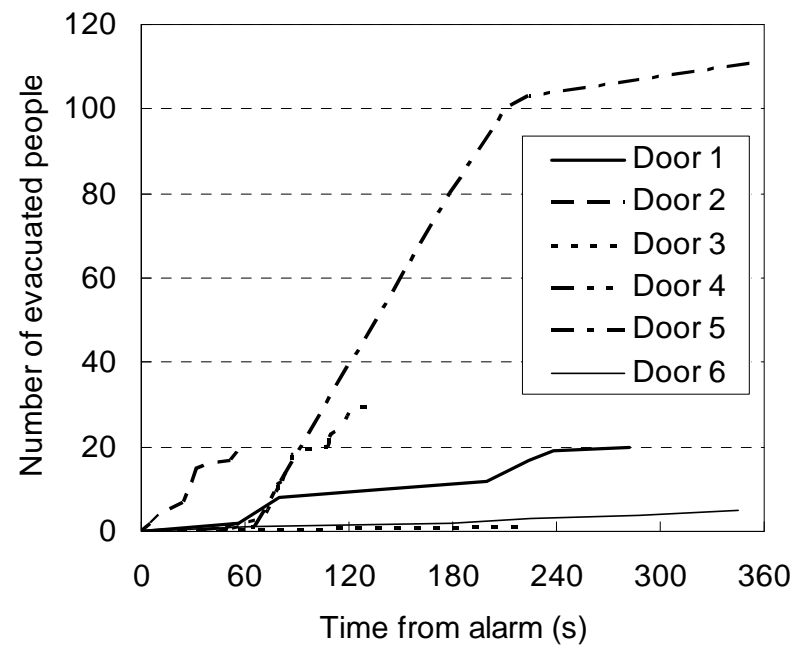

Fig. 5. The cumulative number of evacuated people for the doors of public library.

\section{Large office building}

\section{General observations}

The evacuation exercise was held in November . Before the evacuation, the employees were informed that the exercise would take place at the certain day. Information was printed out on the walls of the elevators.

The smoke generators were turned on simultaneously in staircases 1 and 3. It took about 2 min before the fire alarm went off, and the evacuation started. The first people came out from the staircase 2 in about $1 \mathrm{~min}$ from the alarm. Most of the people came out, as expected, through staircase 2. From doors 1 and 3, 
the first people came out in about $1 \mathrm{~min}$, when smoke was not yet filling all the space. They all belonged to the safety organisation.

The evacuation through staircase 2 was afterwards analyzed using the video recordings. Most of the people walked calmly and no rushing was observed. Queuing was observed, because of merging flows, where some individuals decided to stop and give way for the flow descending from the upper floors. One long queue was once formed when a small group of people coming from the upper floors stopped for $60 \mathrm{~s}$ and allowed the flow from the office to enter the stairway.

\section{Quantitative results}

The virtual control line of the floor levels located just before the first tread going downwards, and the control line of front door located at the doorway. The number of humans originating from a certain floor was counted by summing up the flows coming from the doorways on the left and right sides of the floor landing.

The number of evacuated people through staircase 2 is shown in Fig. 6. On the left side (Fig. 6a) is shown the total flow of people down the stairs at each floor level and right side (Fig. 6b) in turn shows the flow of people entering the stairway at each floor. Total number of evacuated people in 6 min period was 281. The linear parts of the curves show that the human flow was saturated due to the limiting width of the stairs and the front door. Fig. 6b shows that the majority of the people came from the $4^{\text {th }}$ and $1^{\text {st }}$ floors. The number of people using the other doors was: 7 people from door 1 in 4 min, 3 people from door 3 in $50 \mathrm{~s}$ and 2 people from door 4 in 1 min $12 \mathrm{~s}$.

The percentage of RFID tags identified during the test was much better than in the public library. When the number of observations on each floor was compared to the total number of observations of a certain group, it was found that the degree of success was close to $60 \%$. In average, $17 \%$ of the tags delivered were identified in all measurement points. The variation between the groups may be due to the differences between the briefings before the evacuation rehearsal. Clothing may also have an influence. Metallic zippers in the outerwear are made of electrically conducting material.

The average of computed flow rate in staircase for floors 2-4 was 1.0 persons.s. ${ }^{-1}$ and the specific flow was 0.8 persons $\cdot \mathrm{s}^{-1} \cdot \mathrm{m}^{-1}$. For the outdoor $J$ was 1.4 persons $\cdot \mathrm{s}^{-1}$ and $J_{S} 1.3$ persons $\cdot \mathrm{s}^{-1} \cdot \mathrm{m}^{-1}$. For a comparison, the SFPE Handbook [4] lists the maximum specific flow values in different conditions like for a doorway 1.3 persons $\cdot \mathrm{s}^{-1} \cdot \mathrm{m}^{-1}$, which is in good agreement with our results. For stairs, the range is from 0.94 to 1.16 persons s $\mathrm{s}^{-1} \mathrm{~m}^{-1}$ depending on the shape of the tread. Specific flow rates for stairs obtained in our study are slightly lower than the literature values. Possible reasons are the definition of the effective width of the stairs and the fact that in our study, the flow rate was affected by the merging flows from the side doors, especially in the first and fourth floors.
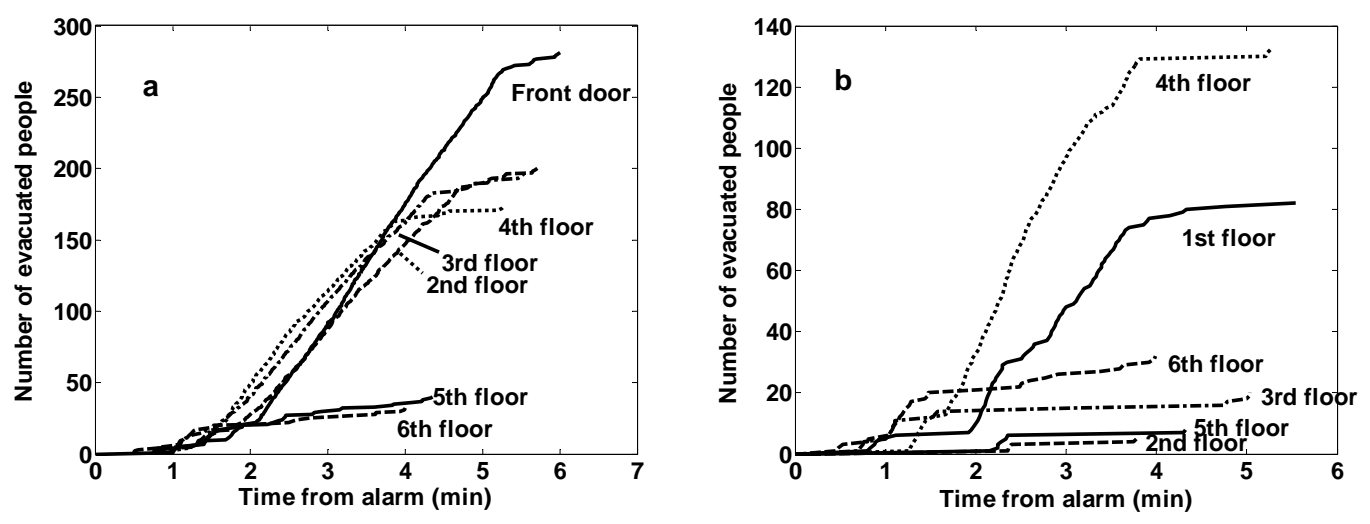

Fig. 6. Number of the evacuated people from staircase: a) accumulated starting from $6^{\text {th }}$ floor and b) the number of people entering the stairway at each floor. 
RFID measurements were used to calculate the exact times spent by individual RFID tagged persons between floor levels. In order to calculate the walking speed, at least two time marks between two floors had to be traced into RFID system.

In the calculation of the crowd flow densities, the local flow rates, shown previously in Fig. 6a, were used at same time as the first time mark to the RFID system was left. This local flow rate was assumed to last until the next RFID observation took place. Thus, the crowd flow density $\rho_{c}$ is expressed in Eq. (3),

$\rho_{c}=\frac{J}{v \cdot w}=\frac{\Delta N}{\Delta t} \cdot \frac{1}{v \cdot w}$,

where $v(\mathrm{~m} / \mathrm{s})$ is the inclined walking speed, which is determined through the distance between the RFID antennas measured using the walking path $l(\mathrm{~m})$ and time interval $\Delta t_{R F I D}(\mathrm{~s})$ between the antennas.

The walking speed on stairs versus crowd density is presented in Fig. 7a. Data points are derived from the traces of the RFID tagged persons in the staircase 2. From Fig. 7a, we can see that, when the crowd density is smaller than 0.5 persons $\mathrm{m}^{-2}$, the evacuees have walked their own walking speed and not because of the descending crowd. At higher densities, the walking speed decreases linearly. The difference between male and female data is not significant. For the descending crowd the overall cumulative distribution function for walking speeds on stairs is shown in Fig. 7b. A lognormal distribution function with parameter $\mu=-0.45$ and $\sigma=0.30$ is fitted to the data points, showing a good correlation. The median of the fitted curve is $0.64 \mathrm{~m} / \mathrm{s}$ and the $95 \%$ fractile is $1.04 \mathrm{~m} / \mathrm{s}$. Comparison to the literature values shows that the current values are slightly lower than those measured by Fujijama \& Tyler in their study [5]. They measured descending inclined walking speed values between $0.76-1.30 \mathrm{~m} / \mathrm{s}$ by changing the stair angle from $24.6^{\circ}$ to $30.6^{\circ}$, corresponding to a qualitative transformation from "normal descending" to "fast descending". In our experiment, the stair angle was about $27^{\circ}$. In the experiments in Ref. [5], the participants were walking alone and not in a crowd, which certainly have an increasing effect to walking speed values. The SFPE Handbook [4] suggests speeds between $0.85-1.05 \mathrm{~m} / \mathrm{s}$ with varying tread shapes. Our result seems to be affected by the queuing effects.

The RFID observations of evacuated people descending in staircase 2 are shown in Fig. 8. These observations were used to estimate the distribution of premovement times in the office building. The times were measured by collecting the first recorded observations of each individual person by the RFID system at $5^{\text {th }}$ and $6^{\text {th }}$ floors. Group 1 and 2 worked at the $6^{\text {th }}$ floor and the members of group 4 at the $5^{\text {th }}$ floor. The first recorded event takes place at $30 \mathrm{~s}$ and the last at $4 \mathrm{~min}$. These are only the time values when a RFID tagged person appeared at the staircase, and the real premovement times are shorter. However, these results can be used to estimate the width of the premovement time distribution.
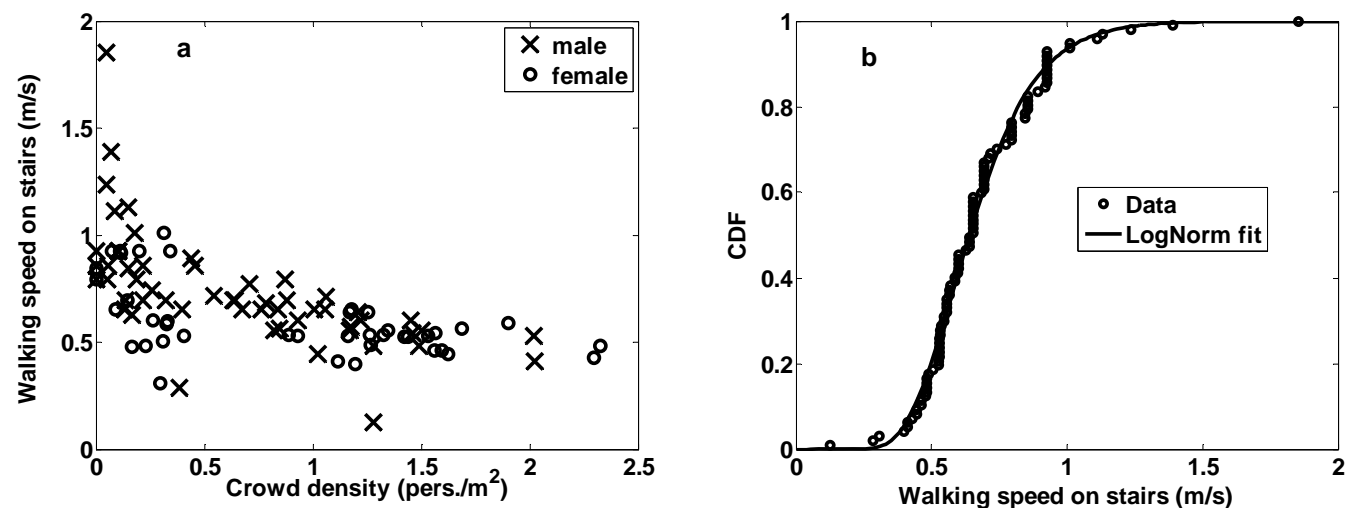

Fig. 7. Walking speed on the stairs of the large office building a) as a function of crowd density and b) cumulative distribution of walking speeds for the crowd. 


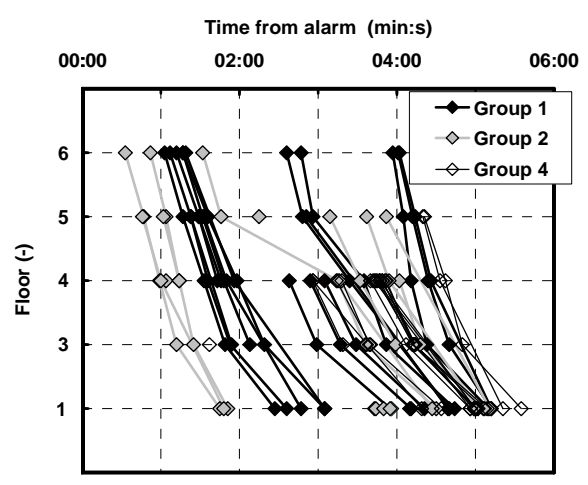

Fig. 8. The recorded times of evacuated people descending in staircase 2. Each single line represents the recorded times of a single person.

Soon after the evacuation, the employees were asked to fill an inquiry form concerning the exercise. The form was on a web site, where the answers were recorded automatically to a database. The inquiry form consisted of 18 questions regarding the different aspects of the evacuation exercise and one open page for free comments (further details of the inquiry form can be found in Ref. [9]). In free comments, where the number of respondents was 19 , several people told they could not clearly hear the fire announcements during the exercise. Also some person would have wanted to have an exercise without any given briefing and information beforehand.

The key findings of the answers (number of respondents was 90) for the staff are the following,

- The briefing and announcements were clearly noticed before the exercise.

- $67 \%$ of respondents had no prior experience on evacuation exercises.

- Almost half of the respondents would have used the main door, the well-known route, to evacuate but in the exercise, doors 2 and 3 were mostly used. This was against our observations. The reason might be that the original alternatives of door selection were given as street addresses, not in a form of "door 1", "door 2". The respondents may have had difficulties to connect the address to a right exit door they used in exercise.

- About 70 persons used the route starting from the staircase 3 and finally ending to door 2 in staircase 2, due to the smoke in staircase 3. This movement could have been interpreted as a use of evacuation route of the staircase 3, which is closer to the street address. According to our bookkeeping, the door 4 was only used by two persons during the whole exercise.

- Selection of the different route was mainly caused by smoke or safety organisation member guidance.

- Office staff felt slight congestion during evacuation in staircase, but it was not conceived as uncomfortable or anxious.

\section{Medium sized office building}

\section{General observations}

The smoke detector in the staircase 2 went off in less then 3 min from the start of the smoke generator. Most of the people came out from the main door (door 5). The door 3, which was assumed to be less familiar, was used extensively due to the guidance from the safety organisation. In point of view of our study, the door selection for observations was not the best possible, because inside the building only few people used the corridor and the staircase 1.

Observations at the staircase 1 revealed how difficult it was to come out from the staircase at the right floor level leading to a right exit, namely the stairs continued down to basement. Two groups of 4 and 7 persons were observed to follow the first person all the way to down the basement, until they noticed that they are 
at wrong floor. After this they had to ascend one floor to reach the corridor and exit. These events took about $30 \mathrm{~s}$ for the first group and $40 \mathrm{~s}$ for the second group.

\section{Quantitative results}

The cumulative sums of evacuated people for each door and the total are shown in Fig. 9. The zero time corresponds to the moment of fire alarm. Most of the people came out from the door 5 (the main door) and the door 3 . Total number of the evacuated people was 139 within approximately 6 min.
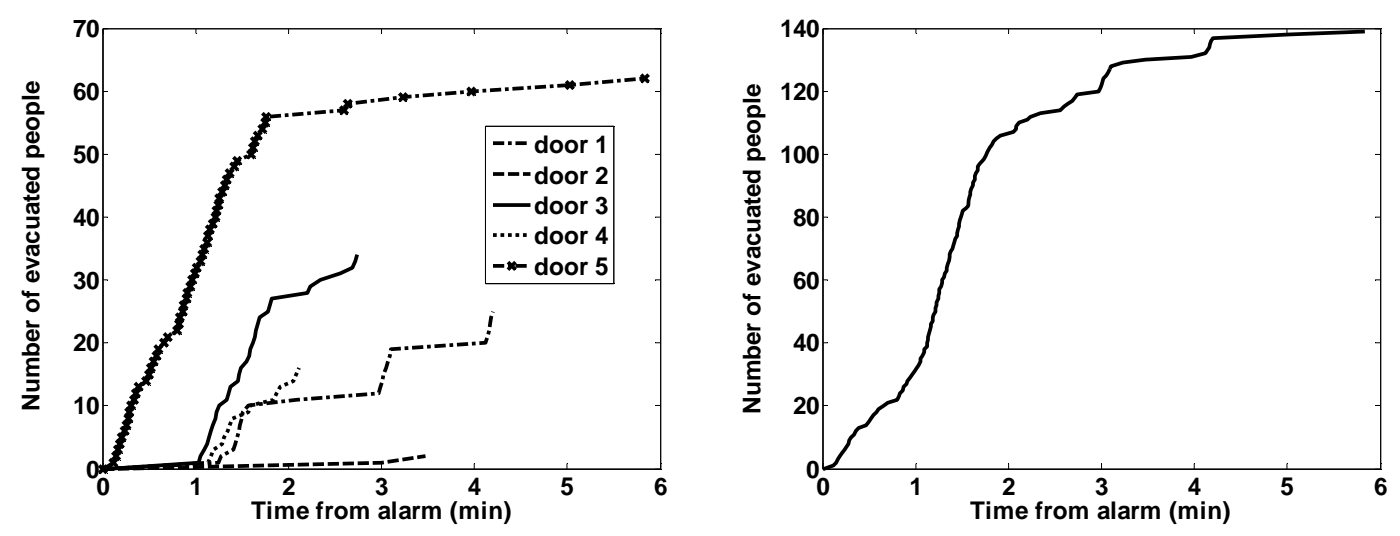

Fig. 9. On the left side number of evacuated persons through different doors and on the right side the total number of evacuated person from the medium sized office building.

The flow rates determined from the Fig. 9 are 0.5 persons/s for the door 3 and 0.58 persons/s for the door 5 . Both values are quite low compared to literature values and our former evacuation cases. By looking the videos, it was seen that the whole capacity of the exit routes was not used. Only 30 people came out through the door 1 and only about 15 of them through the staircase 1 .

The number of successful RFID recordings was only 12 of 50 . The percentage of persons identified by RFID was a little bit better than in the public library evacuation but much smaller than in the large office building. This is mostly due to the fact that many of the test persons did not attend the test. The people entered the staircase 1-3 min from the alarm, which is a little bit faster than in the large office building.

From the inquiry form results, we could see that two thirds of the respondents ( $\mathrm{N}=48$ ) have had some experience of evacuation before and that the evacuation happened mostly by following other personnel and not by following the safety organisation members. The different doors were selected quite uniformly, and the main door was not highlighted, contrary to our observations. The result may be affected by the small number of respondents. It is notable that over half of persons used an exit route that was not well-known for them.

\section{Surveillance cameras as a source of evacuation data}

A large shopping centre (two floors, about $400 \mathrm{~m} \times 120 \mathrm{~m}$ ) in southern Finland had a false fire alarm in February 2007 due to a frozen sprinkler head. At the moment of the alarm, there were more than 1000 customers in the building. The whole building was not evacuated, because there were no other signs of a real fire, and because the weather outside was cold (about $-10^{\circ} \mathrm{C}$ ). The video recordings from four surveillance cameras were obtained. The quality of the video was relatively good, when compared to the surveillance cameras in general, but poorer than the quality of normal digital video cameras.

The human flow corresponding one of the exit doors was counted using a recording of one of the cameras. The camera was monitoring the door from a distance of about $20 \mathrm{~m}$. The exit door had two sliding panes. On both sides of the door, there were doors with latches in the vertical frame. The left and right side doors were opened 128 and $185 \mathrm{~s}$ after the alarm, respectively. The cumulative sum of people that went through the doors is shown on the left in Fig. 10. The people re-entering the building were neglected. The four curves in the figure correspond to different counting positions and different playing speeds of the video 
during the counting. "Line" means a virtual line passing from a corridor wall to wall relatively close to the camera position. "Line/2" means that the line was first divided into two parts, flows for each part were counted separately, and the cumulative results were combined. "Door" means that the virtual counting line was placed at the door line. Terms "fast and "slow" refer to the playing speeds. When using slow playback, more people were observed from the door than when using the fast playback. The curves corresponding the virtual line are between these two "door" curves. The differences in the curves are mainly due to the restricted and steep view angle to the door. Also, the crowd between the camera and door blocked the visibility to the actual door line. These differences can thus be considered as an example of the uncertainty associated with the counting process. The flow rates corresponding to the four counting methods are shown in right side of the figure. Some of the oscillations are probably caused by the numerical derivation process. When the highest peaks are omitted, we can say that the highest flow rate of 1.6 persons $\cdot \mathrm{s}^{-1} \cdot \mathrm{m}^{-1}$ is found in the initial phase of the evacuation. After the opening of the second latch door, the flow rate goes down to 0.6 persons $\cdot \mathrm{s}^{-1} \cdot \mathrm{m}^{-1}$. This is probably result from that the evacuees stopped movement right after they were outside and the crowd was denser more and more at the doorway, thus limiting the crowd flow from inside.
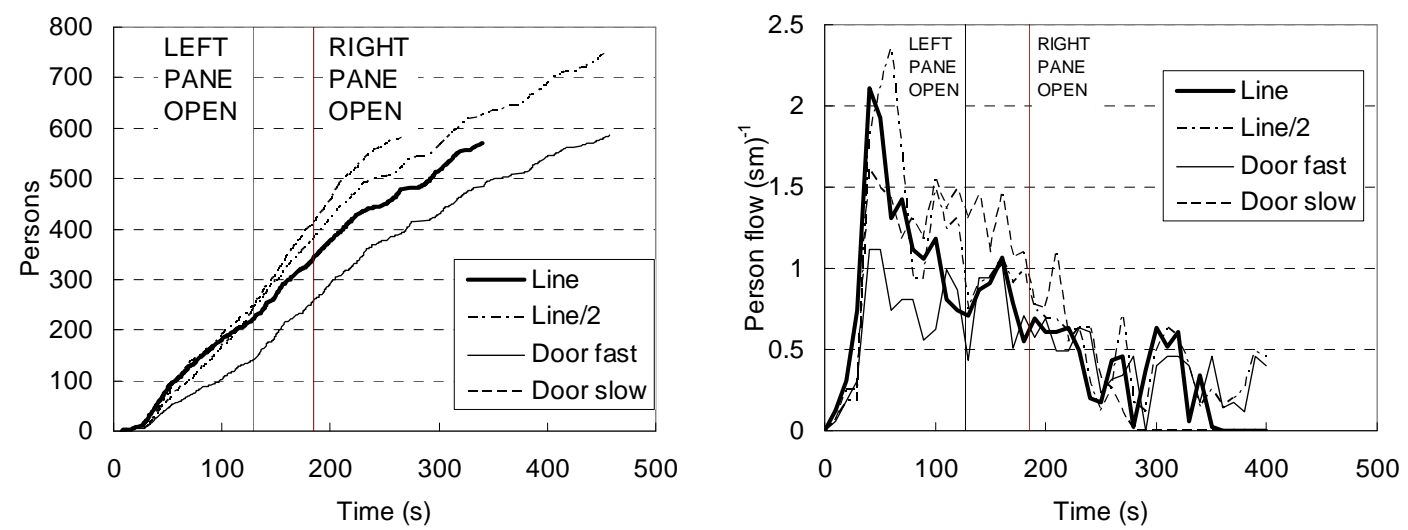

Fig. 10. Flow out of the door during the shopping centre evacuation (left) and flow rate out of shopping centre door (right).

These results demonstrate that the surveillance cameras can be used as a source of detailed information on the evacuation in public spaces. However, the orientation of the cameras is crucial for the reliability of the counting. For good results, a perpendicular and unrestricted view on the evacuation path should be available. These conclusions apply only to the counting of people flows. Observation of reaction and premovement times was not possible from the available videos. In the future, the surveillance cameras may provide a valuable source of information on the realistic behaviour of people in fires on public places.

\section{CONCLUSIONS}

The experimental techniques to observe the human behaviour during evacuation drills were studied in the evacuation of public library and two office buildings. The use of surveillance cameras was studied using the video material recorded during an evacuation of large shopping centre.

Promising results were achieved for the use of RFID as a means to make observations on the human movement in the evacuation. By the careful placement of both the antennas and the tags to be detected, a sufficient reliability for scientific measurements can be achieved. However, the results are very sensitive on the tag placement over or under the clothes, close to metal objects or behind parts of human bodies. The preparation and post-processing of the tag information is still relatively laborious because of human interpretation of the results is needed in the analysis.

The results of the evacuation measurements provide information on the flow rates of people through the doors and stairways, effects of crowd density on the stair flow and distributions of premovement times. All these results can be used both directly as a basis of a building design or as a means to develop and validate the predictive capability of the evacuation simulation. 


\section{ACKNOWLEDGEMENTS}

The support from the safety organizations of the target buildings was essential for the successful implementation of the experiments, and greatly acknowledged. Authors wish to thank the following colleagues for the assistance during the experiments: Ms. Katri Matikainen (Helsinki University), Dr. Pekka Pursula, Mr. Konsta Taimisalo, Mrs. Mari Niemelä, Mrs. Kaisa Belloni, Dr. Kati Tillander, Dr. Esko Mikkola, Mrs. Tuuli Oksanen, Dr. Johan Mangs and Mr. Arto Hätelä of VTT. The work was funded by the Finnish Funding Agency for Technology and Innovation, the Finnish Fire Protection Fund, the Ministry of the Environment, the Academy of Finland and VTT.

\section{REFERENCES}

[1] Purser, D. \& Bensilum, M. Quantification of behaviour for engineering desing standards and escape time calculations. Safety Science. 2001. Vol. 38, pp. 157-182. doi:10.1016/S0925$\underline{7535(00) 00066-7}$

[2] Gwynne, S., Galea, E., Parke, J. \& Hickson, J. The collection of pre-evacuation times from evacuation trials involving a Hospital Outpatient area and a University Library facility. Fire Safety Science-Proceedings of the Seventh International Symposium. International Association for Fire Safety Science. Worcester, MA. 2003. pp. 877-888. doi:10.3801/IAFSS.FSS.7-877

[3] Frantzich, H. Tid för utrymning vid brand. Lund, SE: Brandteknik, Lunds tekniska högskola. 2001. 122 p. ISBN 91 7253-092-8. (in Swedish).

[4] Nelson, H. \& Mowrer, F. Emergency Movement. In: DiNenno, P. J. (Ed.), SFPE. Handbook of Fire Protection Engineering, 3rd Edition. National Fire Protection Association, Quincy MA. 2002. Ch. 14.

[5] Fujiyama, T. \& Tyler, N. An Explicit Study on Walking Speeds of Pedestrians on Stairs. 10th International Conference on Mobility and Transport for Elderly and Disabled People, Hamamatsu, Japan. 2004. 10 p.

[6] Helbing, D. \& Molnár, P. Social Force Model for Pedestrian Dynamics. Physical Review E, 51. 1995. pp. 4282-4286. doi:10.1103/PhysRevE.51.4282

[7] Helbing, D., Farkas, I. \& Vicsek, T. Simulating Dynamical Features of Escape Panic. Nature, 407. 2000. pp. 487-490. doi:10.1038/35035023

[8] Helbing, D., Farkas, I., Molnár, P. \& Vicsek,T. Simulating of Pedestrian Crowds in Normal and Evacuation Situations. In: Schreckenberg, M. and Sharma, S.D. (eds.) Pedestrian and Evacuation Dynamics. Springer, Berlin. 2002. pp. 21-58.

[9] Hostikka, S., Paloposki, T., Rinne, T., Saari, J., Korhonen, T. \& Heliövaara, S. Experimental observations of evacuation situations. Espoo, VTT. VTT Working Papers; 85. 2007. 52 p. 